\title{
Author's reply to discussion on "Hazard assessment of debris flows for Leung King Estate of Hong Kong by incorporating GIS with numerical simulations" by S. Parry, M. E. Ruse and S. J. Williamson
}

\author{
K. T. Chau and K. H. Lo \\ Department of Civil and Structural Engineering, The Hong Kong Polytechnic University, Hung Hom, Kowloon, Hong Kong, \\ China
}

Received: 6 December 2004 - Accepted: 6 December 2004 - Published: 3 January 2005

\section{Introduction}

We would like to express our appreciation to Parry, Ruse and Williamson for their interest in our publication and for providing their opinions on our works. We are happy that the discussion here provides us further opportunity to bring focus on some points relating to our concern on the indiscriminate use of expert opinion in debris flow hazard estimation.

Parry et al. (2005) tried to create an image that we epitomized the use of "numerical approach" against "conceptual approach", accused us of considering "conceptual geological model" being "subjective and biased", claimed that we did not do any field and geological study, and questioned that all of our results are wrong and inaccurate. They further claimed that the use of flow-dynamics-based "numerical approach" is naive, in comparing to the use of "expert opinion". They also argued on a lot of statements that we have never made. We will, therefore, re-iterate what we have actually said, and what are actually misinterpretations resulting from their incorrect deductions from our statements, clear all misunderstanding between us, and conclude that their conclusions are not just. As we spelled out clearly in our work (Chau and Lo, 2004) that we are fully aware of the limitations and assumptions in our "flow-dynamics-based approach". We actually never claim that our conclusion must be correct, and discussions on the limitation of our results are actually welcome. Since the discussions made by Parry et al. (2005) are grouped in paragraphs in their Discussion Section, the following replies try to answer paragraph by paragraph correspondingly.

\section{Replies to discussions}

First of all, we want to make clear that all we have said in the Abstract of Chau and Lo (2004) is that "our proposed hazard

Correspondence to: $\mathrm{K}$. T. Chau

(cektchau@polyu.edu.hk) map was thus determined by flow dynamics and a deposition mechanism through computer simulations without using any so-called expert opinions, which are bounded to be subjective and biased", on Paragraph 1 of Sect. 3.4.1 is that "the advantage of this kind of hazard map is that it is based purely on dynamics, instead of on expert opinions or on past debris flow records", and on Paragraph 2 of Sect. 3.4.1 is that "the main focus here is not on the absolutely accuracy of the hazard estimation, but on the idea of combining the sound theoretical approach in GIS-base hazard mapping, such that expert opinion can be reduced to a minimum". In fact, we have never defined the "so-called" expert opinions because we do not know how to define it accurately and preicsely. The gut feeling of an expert in arriving at the so-called expert opinions is most of the time mysterious and cannot be elaborated lucidly; and making the situation worse different experts with different experiences and backgrounds always arrive at different conclusions for the same set of data (if they are not properly guided). For example, in the subject area of climate change expert opinions are always diversified (Morgan and Keith, 1995). In the study of using expert opinion for assessing seismic hazard in low seismicity area, McGuire (1994) found that "judgment" by experts must be firmly based and justified on a scientific plane, that diversity among experts must be resolved if it is caused by misunderstanding or miscommunications. In addition, these expert opinions have to be quantifiable and documented in order to quantify the associated uncertainty. On studying the landslide risk adjacent to highways, Allison et al. (2004) concluded that there must be a decision framework for experts to follow otherwise consensus from a group of experts cannot be arrived at. Therefore, it is clear that the use or misuse of expert opinions needs to be handled with extreme care. The subjective nature of socalled "expert opinions" are well accepted in the literature. The key issue is how we can reduce such uncertainty to a minimum if there is no other resort to solve the problem. Secondly, we have never said that numerical approach is "more accurate and therefore more scientific than the conceptual ap- 
proach". This statement was made by Parry et al. (2005) only not by us. In fact, the discussers' did not define the so-called "conceptual approach" when it first appeared, therefore, we have no idea what did the discussers mean by "conceptual approach" at that juncture. But, if the discusser asserted that they can estimate the runout of debris flows (that was the main focus of Chau and Lo, 2004) by some kind of conceptual model, without concerning the physics and dynamics of how debris materials flow down gully, we will certainly disagree.

In the second paragraph of the Discussion Section, the discussers "suggest that hazard assessment of debris flows requires a suite of analyses, including evaluation of geology, morphology and process behaviour of the study area and its vicinity". Actually, this is preciously the procedure that we have done in arriving at the necessary information to estimate the input for the modified Takahashi simulation program. As reiterated many times in Chau and Lo (2004), field trips and desk studies have been conducted to study the landslide susceptibility (i.e. identifying the source zones and the corresponding soil properties), before we actually incorporate the flow dynamics model for estimating runout. More details of these field trips are documented by Lin et al. (2003). Therefore, we are totally puzzled by the accusation that we might consider such approach being "expert opinion approach" and being "subjective and biased". As discussion in Chau and Lo (2004), there are of course uncertainties in estimating the input data (location and size of sources) based solely on geological data but such uncertainties can be reduced by a more thorough site investigation, if time and cost allow. Therefore, there are assumptions and simplifications in the process, but we do not see the need of intuition, as asserted by Parry et al. (2005).

We have re-iterated many times in Chau and Lo (2004) that the results of the numerical simulations are not meant for accurate predictions of the debris flow hazard of Leung King Estate, due to the uncertainty in the input. Although our prediction may not be very accurate, at least it is based on physics and dynamics, not the intuition or gut feeling of some experts. Nevertheless, the results of our numerical simulations should roughly reflect the worst scenario. We believe that if we want to prepare for the worst, the debris flow barrier should be longer and higher. Whether we need a longer and higher barrier is a matter of risk analysis in terms of the potential return period of very rare and extreme events, and of cost-benefit analysis. This is, however, out of the scope of Chau and Lo (2004) and out of the scope of this reply as well. The main point here is that we believe the most accurate way of estimating the runout (i.e. the hazard of debris flow) is to use the known physics and dynamics of flowing debris if the total volume can be estimated, not expert opinions. In fact, we suspect that the discussers do not have any better counter-proposal to estimate the runout of debris flow if the total volume is known. I guess, that is the reason why they do not offer a better option here.

It is difficult to understand why the discussers keep saying that we fail to consider the historical and geomorphologi- cal data. As discussed in Lin et al. (2003) and also in our early studies on rockfall (Chau et al., 2003; 2004b) and landslides (Chau et al., 2004a), we always consider historical and geomorphological data before conducting any kind of analysis. As pointed out correctly by the discussers, the main uncertainties in our flow-dynamics-based numerical simulations lie on setting up of the hydrograph of the debris flow (flow rate versus time), and on the estimation of the total volume of debris that may be channelized. But for those "short" gullies found in the west of Leung King Estate, the hydrograph is expected come in one surge (instead of series of surges for gullies of tens of kilometers long), and it was found that the shape of hydrograph from Mount St. Helen debris flow closely resembles those observed elsewhere for the case of short gullies (Lo, 2003; Takahashi, 1991). In fact, we maintain the shape of the hydrograph of the Mount St. Helen debris flow, not the velocity as claimed by Parry et al. (2005). Regarding the volume, as we mentioned earlier we are looking at the worst scenario or the most extreme case. The discussers claimed that their designed $600 \mathrm{~m}^{3}$ were reviewed and considered to be appropriate to protect the Estate from future failure. Unfortunately, the discussers did not share with us how they review and consider this value to be appropriate (I sincerely hope it is not done by expert opinions).

Regarding the modification to Takahashi et al.'s (1992) model, we are adopting the concept of soil entrainment and re-entrainment by overland flow using the concept of stream power of flow commonly adopted in the area of hydrology (Marshall et al., 1996). In fact, the entrainability of substrate has already been incorporated in Eq. (6) through the use of particle size distribution of the substrate. The use of settling of velocity in Eq. (6) is simply to reflect the fact that flow containing high soil concentration cannot pick up any more soil (or no entrainment). The full details of this concept are, however, referred to Marshall et al. (1996) due to space limitation. In addition, the local slope gradient has also been incorporated into the present analysis. As discussed by Chau and Lo (2004), the topographical model needed by the analysis of Takahashi was generated by the DEM (or so-called TIN in ArcGIS), and the model by Takahashi is formulated to simulate flows on three-dimensional terrain. Therefore, the effects of entrainability and local slope gradient are both embedded in the numerical simulation.

The discussers are correct that the landslide susceptibility of Evans et al. (1999) did not include the runout estimation. In fact, there is no other published result on the estimating the runout at Leung King Estate, that is the reason why we compare our results with those predictions based purely on the susceptibility analysis of Evans et al. (1999). The key issue here again is that expert opinion approach cannot yield any estimate of runout, and clearly the discussers agreed with us that runout distance of debris flow must be incorporated into the debris flow hazard analysis.

In the last paragraph, the discussers described what might have happened during the 1990 Tsing Shan debris flow (King, 1996). The discussers tacitly asserted that the mor- 
phological setting at the site of 1990 Tsing Shan debris flow favored debris entrainment while that of Leung King area limits entrainment. They attributed this argument to the reference of Fletcher et al. (2002). Again, unfortunately, they failed to pinpoint exactly what geological conditions that limit entrainment at Leung King while what are those favor entrainment at the 1990 debris flow site. In fact, Fletcher et al. (2002) included both areas as one whole study area and discussed their geomorphological evolution in one unit. There are no discussion on the differences of the morphological settings between Leung King and the 1990 debris flow site. Even if there are topographical differences as well as differences in entrainable materials, the present analysis of Chau and Lo (2004) can handle these changes easily. The local slope gradient are automatically taken into consideration by the DEM model from GIS from one gully to another, while local soil properties can always be obtained from site investigation such that the particle size distribution of the discharge as well as the substrate can be modified accordingly.

\section{Conclusion}

With all of the above discussions, we found that simply claiming that "the disadvantage of Chau and Lo's approach is that "it is based on dynamics, instead of expert opinions" is unfounded and unsubstantiated. It is also puzzling that if the physics and dynamics of the flow debris can be formulated based upon Newtonian mechanics, why we still want to make decision based solely on expert opinions. As mentioned in McGuire (1994), expert opinions need to be used only if there is no reliable data, no reliable method and no reliable approach for estimating hazard. The so-called "conceptual approach" has in fact been used when the input data for numerical simulation was formulated, and for the final prediction of runout flow-dynamics-based approachb. Therefore, we think that the present approach is a natural step forward and numerical simulation need to be included in hazard analysis, and do not see why such approach is "naive". The discussers also claimed that our conclusion that numerical simulation results must be incorporated "is not supported by the case presented". Again, the discussers were not generous enough to offer the specific argument why it is not supported in this case. Probably they assumed that their claim that a total volume of $600 \mathrm{~m}^{3}$ must be appropriate and anything larger than that must be wrong. While we fully aware and accept the possibility that our estimate of the flow volume may be in error, they appear to refuse accepting the chance that their estimate may also be in error (especially when there is no scientific basis for the absolute correctness for the value of $600 \mathrm{~m}^{3}$ ). Whether the future debris flow events at Leung King Estate will be less than $600 \mathrm{~m}^{3}$ or substantially larger, time will tell. Let us be open-minded in dealing with the future. Despite all these disagreements, we totally agree with the discussers that good judgment (not expert opinions) is extremely important even in our numerical analysis.
Nevertheless, we wish to thank Parry et al. for initiating this discussion which, we are sure, should provide stimulus for future scientific research and progress in this area of high uncertainty.

Edited by: F. Guzzetti

\section{References}

Allison, C., Sidle, R. C., and Tait, D.: Application of decision analysis to forest road deactivation in unstable terrain, Env. Manag., 33, 2, 173-185, 2004.

Chau, K. T. and Lo, K. H.: Hazard assessment of debris flow for Leung King Estate of Hong Kong by incorporating GIS with numerical simulations, Nat. Haz. Earth Sys. Sc., 4, 1, 103-116, 2004, SRef-ID: 1684-9981/nhess/2004-4-103.

Chau, K. T., Sze, Y. L., Fung, M. K., Wong, W. Y., Fong, E. L., and Chan, L. C. P.: Landslide hazard analysis for Hong Kong based on landslide inventory and GIS, Computers and Geosciences, 30, 429-443, 2004a.

Chau, K. T., Tang, Y. F., and Wong, R. H. C.: GIS based rockfall hazard map for Hong Kong, International Journal of Rock Mechanics and Mining Science, 41, 3, 530, 2004b.

Chau, K. T., Wong, R. H. C., Lui, J., and Lee, C. F.: Rockfall hazard analysis for Hong Kong based on rockfall inventory, Rock Mechanics and Rock Engineering, 36, 5, 383-408, 2003.

Fletcher, C. J. N., Massey, C. I., Williamson, S. J., and Parry, S.: Importance of bedrock and regolith mapping for natural terrain hazard studies: an example from the Tsing Shan area, Hong Kong, Natural Terrain-A constraint to development? IMM, Hong Kong Branch, 61-75, 2002.

King, J. P.: The Tsing Shan debris flow, Special Project Report SPR 6/96, Hong Kong: Geotechnical Engineering Office, Civil Engineering Department, 1996.

Lin, H. Y., Chau, K. T., Lee, C. M., Lo, K. H., and Wong, R. H. C.: Landslide evidences and geological history of the northeastern flank of Tsing Shan, International Conference on Slope Engineering, edited by Lee, C. F. and Tham, L. G., 405-410, 2003.

Lo, K. H.: Numerical simulations of debris flow and their application to hazard mapping using GIS, MPhil. Dissertation, Department of Civil and Structural Engineering, The Hong Kong Polytechnic University, Hong Kong, 2003.

Marshall, T. J., Holmes, J. W., and Rose, C. W.: Soil Physics, $3^{\text {rd }}$ edition, Cambridge University Press, 1996.

McGuire, R. K.: Using expert opinion for assessing seismic hazard in low-seismicity areas, Geologie En Mijnbouw, 73, 2-4, 407414, 1994.

Morgan, M. G. and Keith, D. W.: Climate-change - subjective judgments by climate experts, Environmental Science \& Technology, 29, 10, A468-A476, 1995.

Parry, S., Ruse, M. E., and Williamson, S. J.: Discussion on "Hazard assessment of debris flows for Leung King Estate of Hong Kong by incorporating GIS with numerical simulations", Nat. Haz. Earth Sys. Sc., 5, 23-24, 2005,

SRef-ID: 1684-9981/nhess/2005-5-23.

Takahashi, T.: Debris flow, International Association for Hydraulic Research, Balkema, Rotterdam, 1991.

Takahashi, T., Nakagawa, H., Harada, T., and Yamashiki, Y.: Routing debris flows with particle segregation, J. Hydr. Engin., 118, 11, 1490-1507, 1992. 